\title{
Design of Non-Contact Infra-Red Thermometer Based on the Sensor of MLX90614
}

\author{
Gang Jin ${ }^{1, *}$, Xiangyu Zhang ${ }^{2}$, Wenqiang Fan ${ }^{1}$, Yunxue Liu ${ }^{1}$ and Pengfei $\mathrm{He}^{1}$ \\ ${ }^{1}$ Institute of Optical-electronic Engineering, Yantai University, Yantai, Shandong, China \\ ${ }^{2}$ Tianjin Huaning Electronics Co., Ltd. Tianjin, China
}

\begin{abstract}
Liquid identification becomes more and more important in the safety inspection at the subway, airport, and railway. Non-contact liquid security identification is the best way, because it can avoid the contamination of the liquids and the injuries caused by some corrosive and toxic liquids. The paper designed the non-contact Infra-Red thermometer based on the sensor of MLX90614 and the most favored microprocessor STM32F107 for the non-contact liquid security identification system. Completed the hardware circuit, made the PCB plate, programmed the software with Keil C and debugged by Keil uVision4 MDK V4.22. Then, studied the effects by measuring the temperature differences using bottles with various shapes, materials, sizes and wall thickness at different distances. The system meets the requirements of high reliability, low costs, low power consumption, real time response and the demands of the non-contact liquid security identification system.
\end{abstract}

Keywords: Digital sensors, Infra-Red thermometer, MLX90614, Safety inspection, STM32F107.

\section{INTRODUCTION}

Liquid identification becomes more and more important in the safety inspection at the subway, airport, ferry, railway, and so on. Non-contact liquid security identification is the best way because it can avoid the contamination of the liquids and the injuries caused by some corrosive and toxic liquids. It can also speed up the identification. Yet the accuracy of the non-contact liquid security identification is affected by the temperature and it is important to measure the temperature of the liquid when identified with microwave equipment.

There are many ways to measure the temperature of objects. The temperature measurement can be mainly divided into contact measurement and non-contact measurement. We choose the non-contact method based on the infrared technology [1] to measure the temperature for the liquid in the bottle, especially in cases of fast, accurate, and closed bottles.

This paper designs the non-contact Infra-Red thermometer based on the sensor of MLX90614 and the most favored microprocessor STM32F107, and studies the hardware construction and the software design in details. This software is programmed with Keil C and debugged by Keil uVision4 MDK V4.22, and studies the effect of the temperature on different bottles with different shapes and materials. Also, it corrects the results of the measured temperature. It meets the requirements of high real-time, reliable,

\footnotetext{
*Address correspondence to this author at the Qingquan Road, Yantai, Shandong, China. Postcode: 264005; Tel: 08613780951616 ; E-mail: jingang107@126.com
}

low cost and low power consumption. The system works efficiently.

\section{HARDWARE DESIGN}

\subsection{Structure of the Non-Contact Infra-Red Thermome- ter}

The temperature measurement system mainly acquires the temperature of liquids and stores the temperature data into flash memory for system use, and can display the data on LCD. The user can also set the upper and lower limits by keys module. The system gives the audio-light alarm if it goes beyond the limits. Structure diagram of temperature measurement system is shown in Fig. (1).

\subsection{Microprocessor STM32F107}

The microprocessor is the central processing unit of the temperature measurement system. STM32F107 is a kind of ARM Cortex ${ }^{\mathrm{TM}}$-M3 RISC [2] 32-bit processor with produced by STMicroelectronics. The maximum frequency of the processor can reach up to $72 \mathrm{MHz}$ and the flash memory is 64 to 256 Kbytes [3]. It has high integration level, high-performance and low power consumption.

Structure diagram of STM32F107 connectivity line LQFP100 pin out is shown as Fig. (2).

\subsection{Digital Thermometer MLX90614}

The MLX90614 is an Infra-Red thermometer for noncontact temperature measurements which is manufactured by Melexis. 


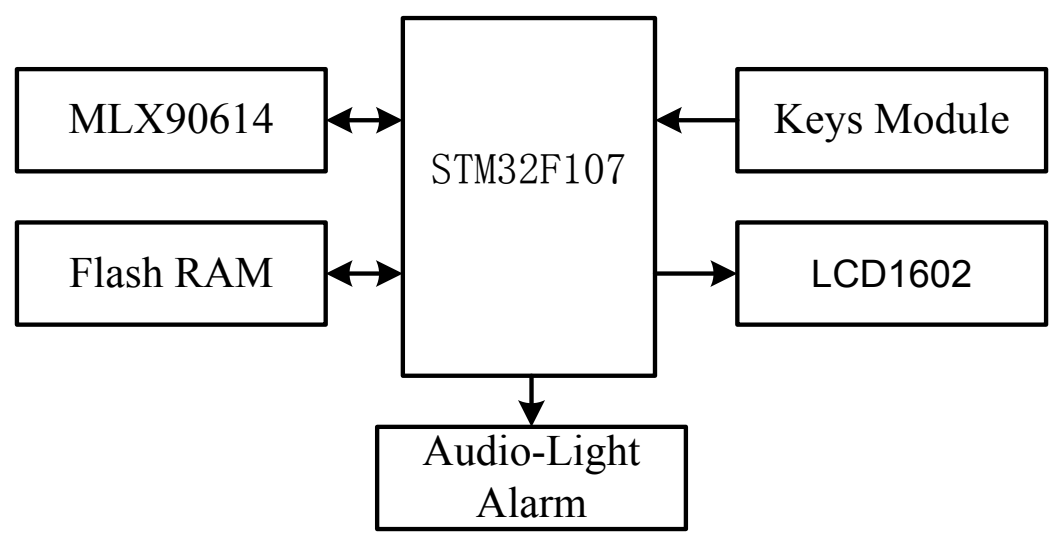

Fig. (1). Structure diagram of temperature measurement system.

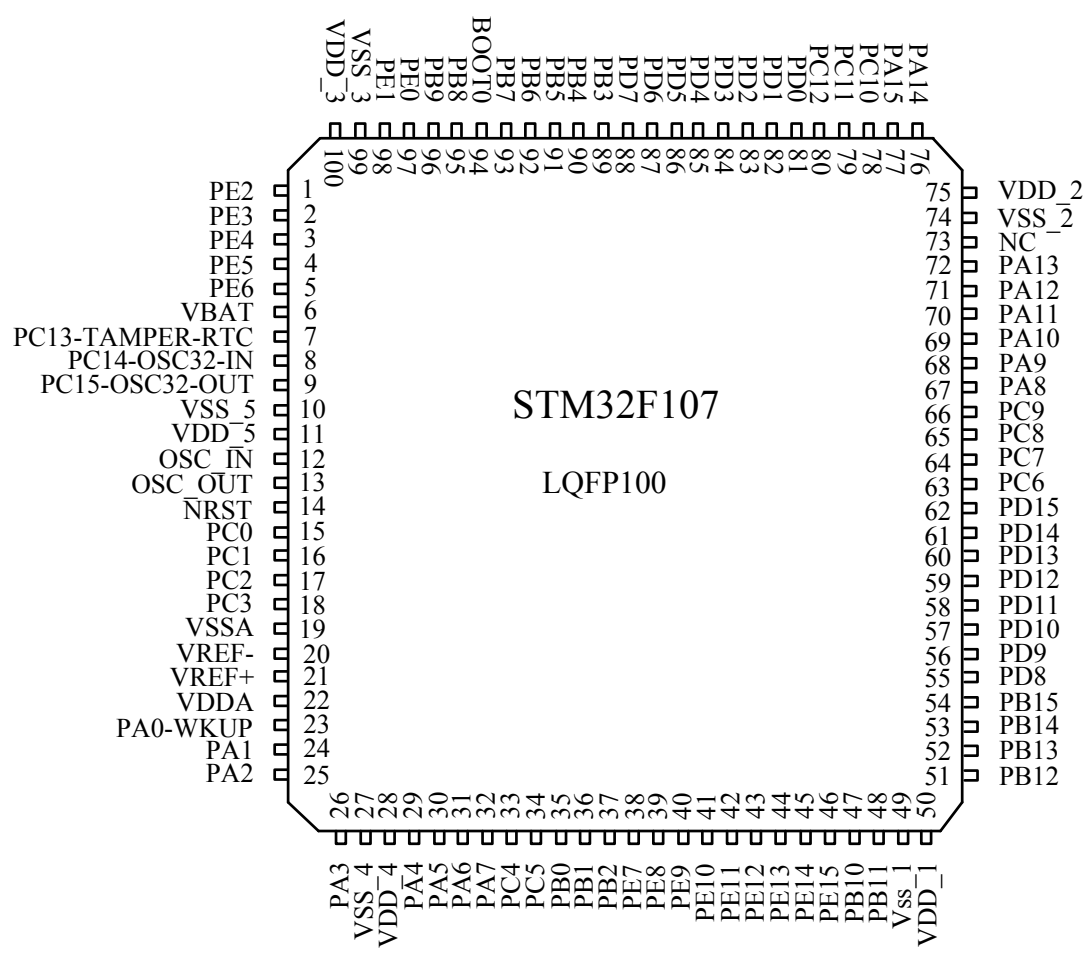

Fig. (2). Structure diagram of STM32F107 connectivity line LQFP100 pin out.

Due to its low noise amplifier, 17-bit ADC and powerful DSP unit, a high accuracy and resolution of the thermometer is achieved. The thermometer used is factory calibrated with a digital PWM and SMBus (System Management Bus) output. As a standard, the 10-bit PWM is configured to continuously transmit the measured temperature in the range of -20 to $120{ }^{\circ} \mathrm{C}$ with an output resolution of $0.14{ }^{\circ} \mathrm{C}$ [4].

Pin function of MLX90614 is shown as Table 1, and pin description is shown as Fig. (3).

In normal mode the measured object temperature is available at pin 2 with Pulse Width Modulated. And in SMBus [5] compatible mode is automatically configured as open drain NMOS.

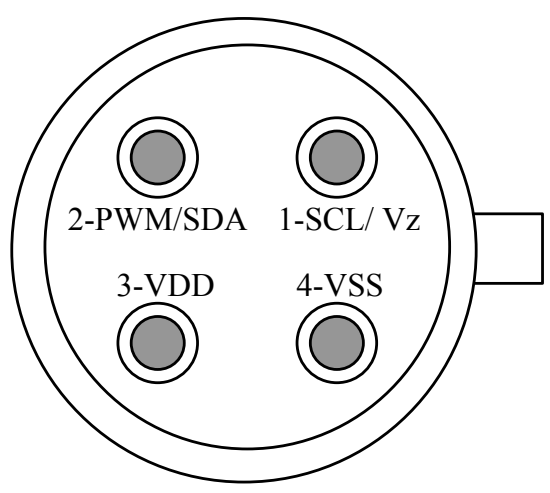

Fig. (3). Pin description of MLX90614 (Top view). 
Table 1. Pin functions of MLX90614.

\begin{tabular}{|c|c|c|}
\hline Pin & Name & Pin Function \\
\hline \hline 1 & SCL & Serial Clock Input \\
\hline 2 & PWM/SDA & Data In/Out \\
\hline 3 & VDD & External Supply Voltage \\
\hline 4 & VSS & Ground \\
\hline
\end{tabular}

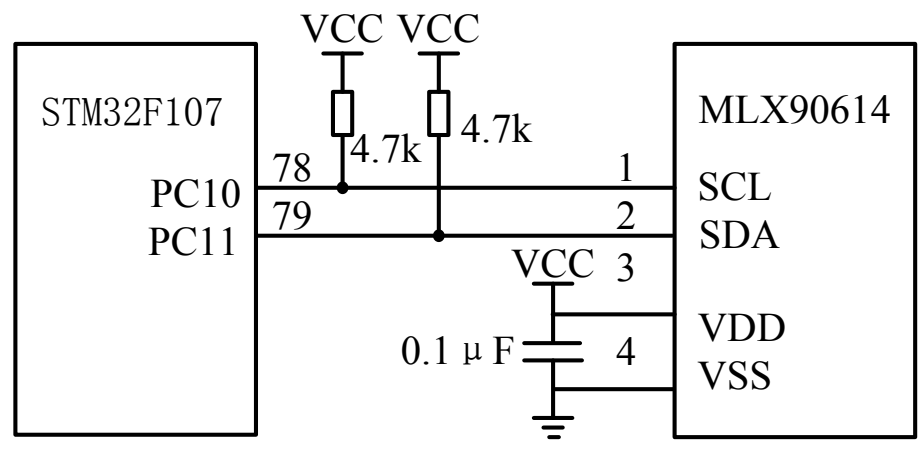

Fig. (4). Structure diagram of STM32F107 and MLX90614 connection.

Table 2. Pin function of LCD1602.

\begin{tabular}{|c|c|c|}
\hline Pin & Name & Pin Function \\
\hline \hline 1 & VSS & PND \\
\hline 2 & VDD & Contrast Adjust \\
\hline 3 & VO & Register Select \\
\hline 4 & RS & Read/Write \\
\hline 5 & R/W & Enable Signal \\
\hline 6 & E & Data Bit $0 \sim 7$ \\
\hline $7 \sim 14$ & DB0 DB7 & Backlight Positive Pole \\
\hline 15 & A + & Backlight Negative Pole \\
\hline
\end{tabular}

The structure diagram of STM32F107 and MLX90614 connection is shown as Fig. (4).

\subsection{Displayer Module}

Displayer module can display the temperature data of the object. LCD1602 is a kind of display which can display $16 * 2$ characters with low power consumption. Pin function of LCD1602 is shown as Table 2.

The structure diagram of STM32F107 and LCD1602 connection is shown in Fig. (5).
The VO pin connects a $10 \mathrm{~K}$ variable resistor as contrast adjusts. A+ connects $+3.3 \mathrm{~V}$ as backlight positive of power supply and K-connects GND as backlight cathode of power supply.

\subsection{Keys Module}

Six keys module is designed as a part of a friendly human-machine interaction interface. The definitions of keys are shown as Table 3. Key 1 together with Key $2 \sim$ Key 3 can set the up/down limits of alarm value. 


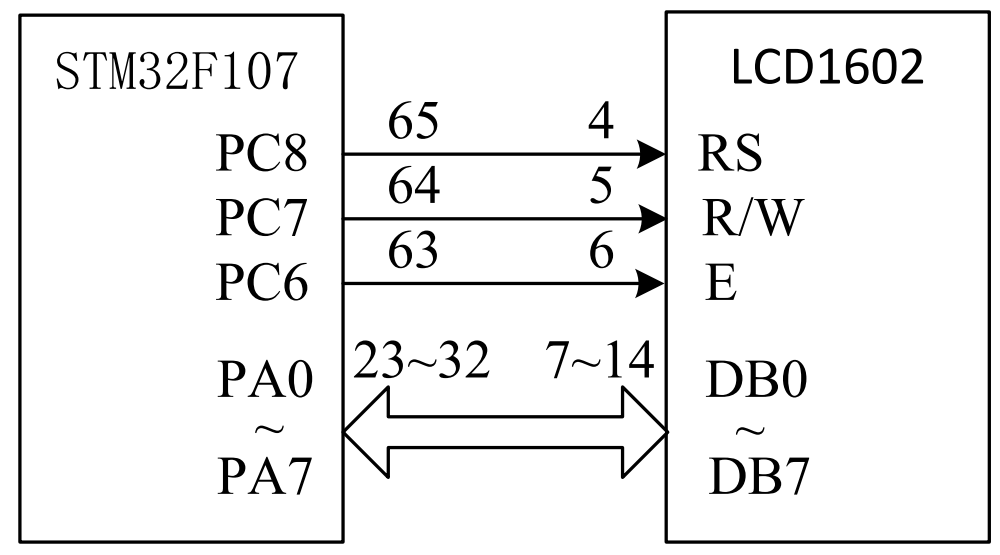

Fig. (5). Structure diagram of STM32F107 and LCD1602 connection.

Table 3. Key definitions.

\begin{tabular}{|c|c|c|}
\hline Key & Name & Definition \\
\hline \hline 1 & Set & Set Temperature Alarm Value Limit \\
\hline 2 & Shift & A Circular Left Shift \\
\hline 3 & Increase & The Value Plus One \\
\hline 4 & Measure & Cancel the Modified Data Before \\
\hline 5 & Cancel & Acknowledgement Key \\
\hline 6 & OK & \\
\hline
\end{tabular}

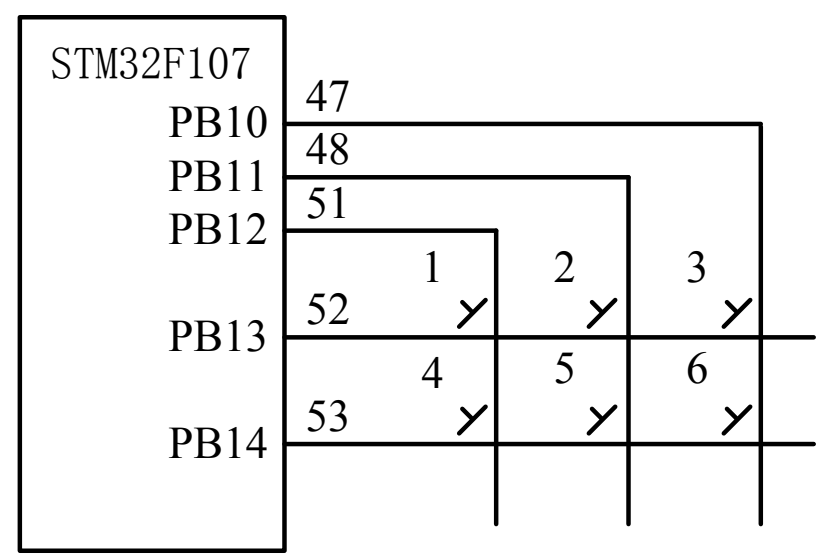

Fig. (6). Structure diagram of key module.

These six keys are organized as a 2 by 3 matrix with 5 ports of STM32107. The structure diagram of key module is shown in Fig. (6).

\subsection{Other Modules}

The non-contact Infra-Red thermometer module uses the 4GB flash memory K9LBG08U0M made by Samsung for data storage. The audio-light alarm system is configured with a beeper and red LED. The alarm sets off if the meas- ured value goes beyond the limits. It uses the DC power and the battery power to ensure the reliability. The system can also work in sleep mode to reduce power consumption.

\section{SOFTWARE DESIGN}

\subsection{The Transport Protocols of MLX90614}

The MLX90614 measures and calculates the temperature of the object and provides the results through the PWM out- 


\begin{tabular}{|c|l|l|l|l|l|l|}
\hline $\mathrm{S}$ & Slave Address & Wr & A & Data Byte & A & P \\
\hline $\mathrm{S}$ & Start Condition \\
$\mathrm{Wr}$ & Write(bit value of 0) \\
$\mathrm{A}$ & Acknowledge (can be 0 for ACK and 1 for NACK) \\
$\mathrm{P}$ & Stop Condition \\
$\square$ & Slave-to-Master \\
$\square$ & Master-to-Slave \\
\hline
\end{tabular}

Fig. (7). SMBus packet element key.

\begin{tabular}{|c|c|c|c|c|c|c|c|c|c|c|c|c|}
\hline 1 & 7 & 1 & 1 & 8 & & 1 & 1 & & 7 & 1 & 1 & \multirow[b]{2}{*}{ ・・・ } \\
\hline \multirow[t]{3}{*}{ S } & $\begin{array}{c}\text { Slave } \\
\text { Address } \\
\end{array}$ & $\mathrm{Wr}$ & A & \multicolumn{2}{|c|}{ Command } & A & $\mathrm{Sr}$ & & $\begin{array}{l}\text { lave } \\
\text { ddress }\end{array}$ & $\mathrm{Rd}$ & A & \\
\hline & & & \multicolumn{2}{|r|}{8} & 1 & \multicolumn{3}{|c|}{8} & 1 & 8 & 1 & 1 \\
\hline & & .. & \multicolumn{2}{|c|}{$\begin{array}{c}\text { Data Byte } \\
\text { Low }\end{array}$} & A & \multicolumn{3}{|c|}{$\begin{array}{c}\text { Data Byte } \\
\text { High }\end{array}$} & A & PEC & A & $\mathrm{P}$ \\
\hline
\end{tabular}

(a) SMBus Read Word Format

\begin{tabular}{|c|c|c|c|c|c|c|c|c|c|c|c|}
\hline & 1 & 7 & 1 & 1 & \multicolumn{2}{|c|}{8} & 1 & & & & \\
\hline $\mathrm{S}$ & $\mathrm{S}$ & $\begin{array}{c}\text { Slave } \\
\text { Address }\end{array}$ & $\mathrm{Wr}$ & A & \multicolumn{2}{|c|}{ Command } & A & \multicolumn{2}{|c|}{$\cdots \bullet$} & & \\
\hline & & & & 8 & 1 & & 8 & 1 & 8 & 1 & 1 \\
\hline & & ‥ & $\begin{array}{r}\text { Dat } \\
\text { I }\end{array}$ & $\begin{array}{l}\text { Byte } \\
\text { ow }\end{array}$ & A & $\begin{array}{r}\text { Dat } \\
\mathrm{H}\end{array}$ & $\begin{array}{l}\text { Byte } \\
\text { igh }\end{array}$ & A & PEC & A & $\mathrm{P}$ \\
\hline
\end{tabular}

(b) SMBus Write Word Format

$\begin{array}{ll}\mathrm{S} & \text { Start Condition } \\ \mathrm{Sr} & \text { Repeated Start Condition } \\ \mathrm{Rd} & \text { Read(bit value of 1) } \\ \mathrm{Wr} & \text { Write(bit value of } 0 \text { ) } \\ \mathrm{A} & \text { Acknowledge }\end{array}$

P Stop Condition

PEC Packet Error Code

$\square \quad$ Master-to-Slave

Slave-to-Master

Fig. (8). SMBus read/write word format.

put or the SMBus interface which are built with pins PWM/SDA and SCL [6]. The paper uses the SMBus protocol.

The SMBus interface is a 2-wire protocol, allowing communication between the Master Device (MD) and one or more Slave Devices (SD). At a given time, only one master device can be present and the MLX90614 can only be used as a slave device.
SMBus packet element key is shown as Fig. (7).

SMBus read/write word format is shown as Fig. (8).

The flow charts of SMBus Write/Read Word is shown in Fig. (9).

The flow charts of temperature measurement by MLX90614 is shown as Fig. (10). 




(a) SMBus Write Word

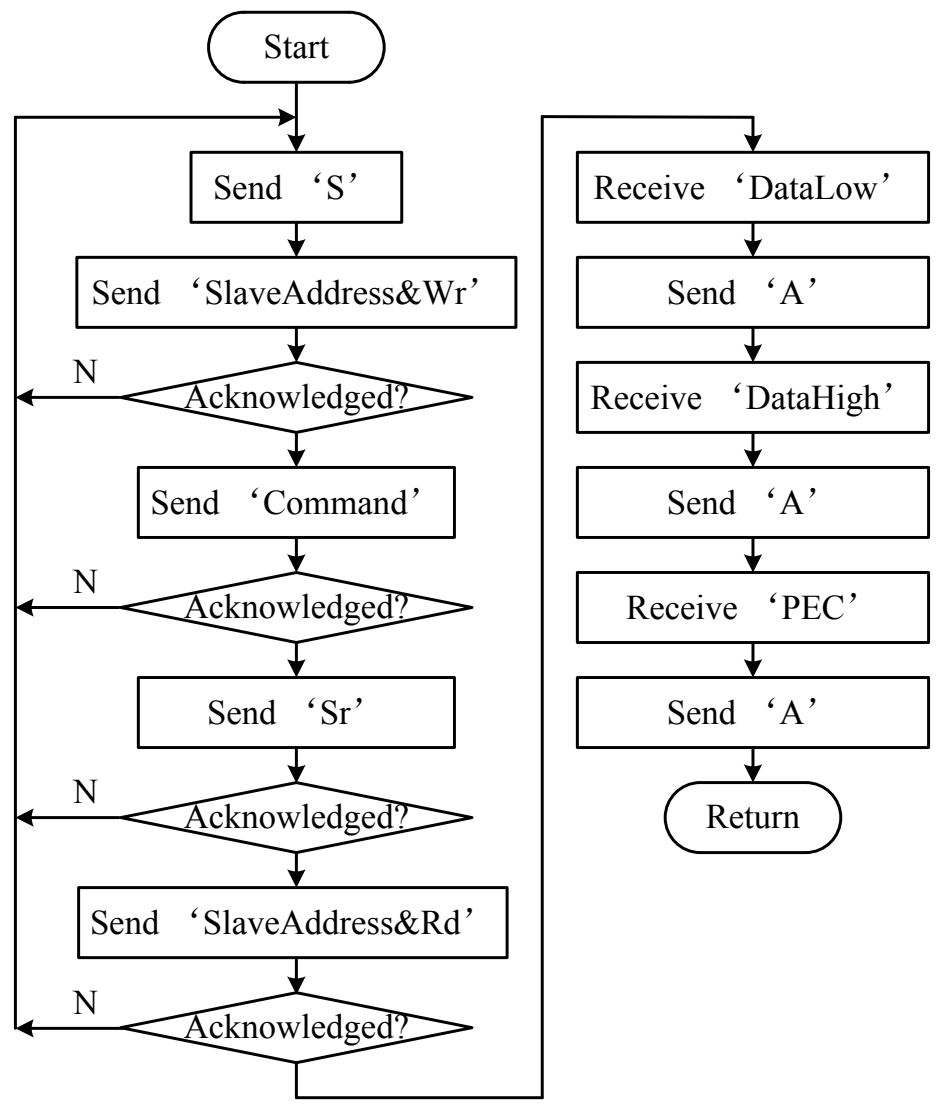

(b) SMBus Read Word

Fig. (9). The flow charts of SMBus write/read word. 


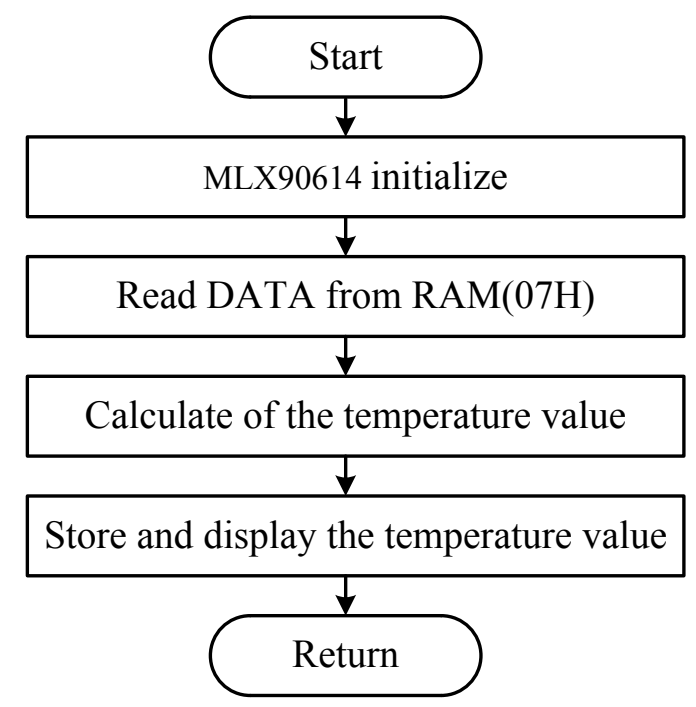

Fig. (10). The flow charts of temperature measurement by MLX90614.

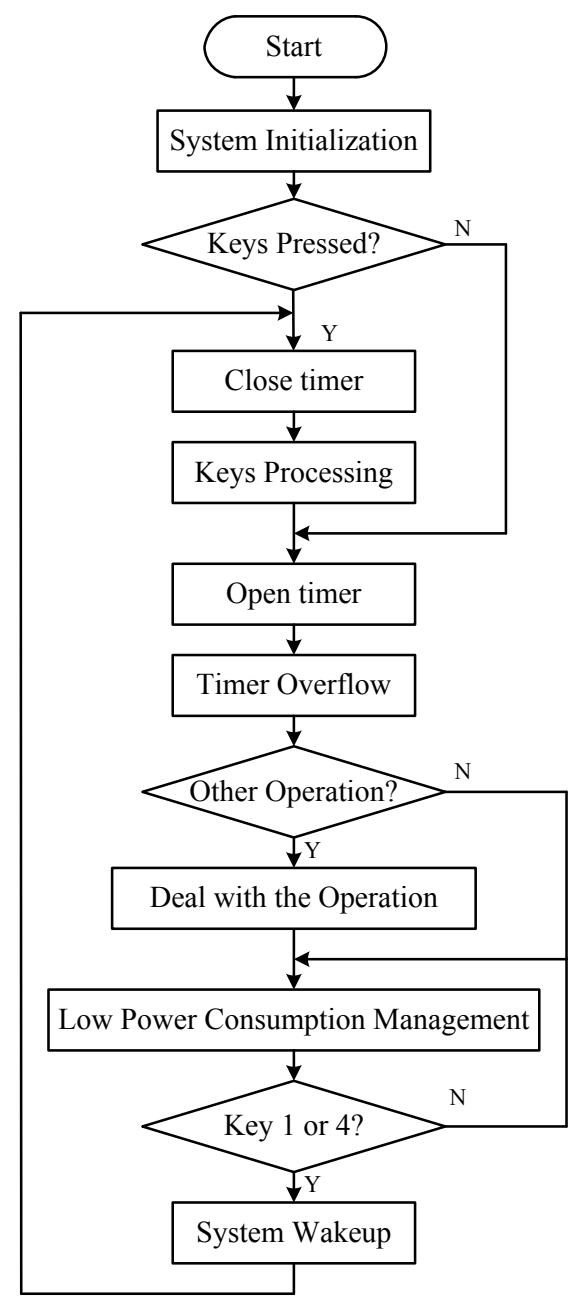

Fig. (11). The flow chart of main program.

It can get the Celsius temperature from the formula $T\left({ }^{\circ} \mathrm{C}\right)=[$ DataHigh : DataLow $] * 0.2-273.15$

\subsection{Main Program}

Main program is a big loop. The flow chart of main program is shown in Fig. (11). 
Table 4. The distance coefficient requirement.

\begin{tabular}{|c|c|c|c|c|}
\hline $\mathbf{D}(\mathbf{m m})$ & $\mathbf{1 0}$ & $\mathbf{2 0}$ & $\mathbf{5 0}$ & $\mathbf{1 0 0}$ \\
\hline \hline $\mathrm{S}(\mathrm{mm})$ & $<120$ & $<240$ & $<600$ & $<1200$ \\
\hline
\end{tabular}

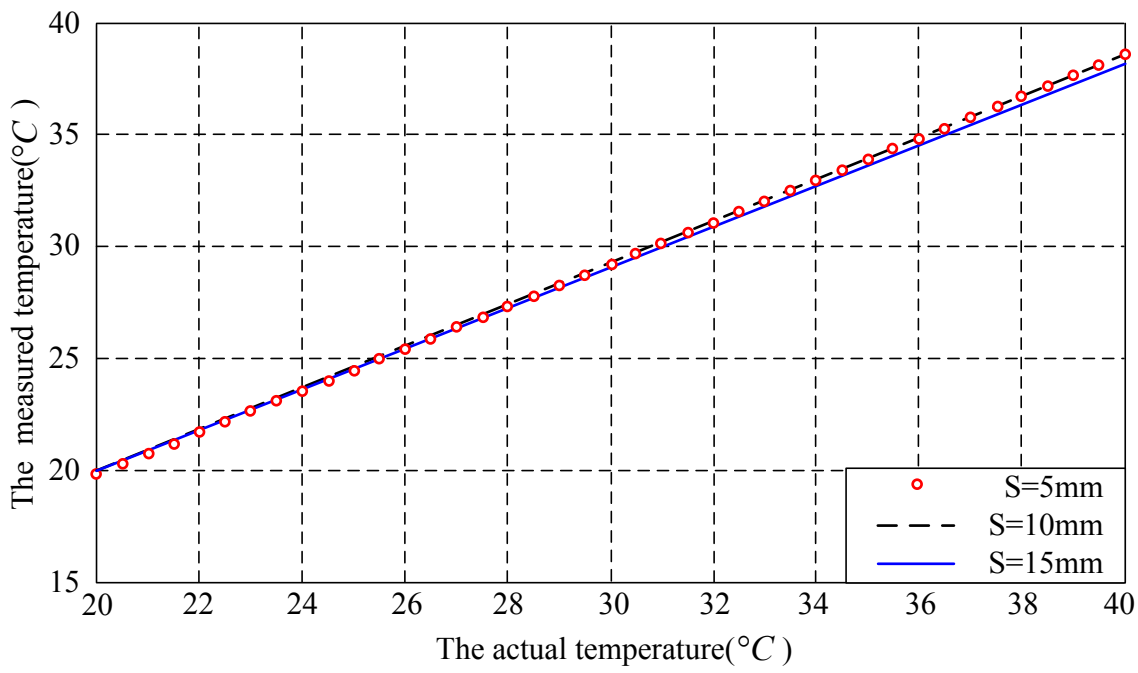

Fig. (12). The comparison of the measured temperature to the actual temperature of the commercially available plastic water bottle at different distances.

\section{ANALYSIS OF THE TEST RESULTS}

The measurement results of liquid temperature are affected by many factors such as distance coefficient, measured distances, the material, the shape and the thickness of liquid bottle. It is necessary to study these effects to eliminate the measurement error. Considering the working environment of liquid security identification system, the test temperature is from $20^{\circ} \mathrm{C}$ to $40^{\circ} \mathrm{C}$.

\subsection{Distance Coefficient}

The distance coefficient $\mathrm{S} / \mathrm{D}$ is one of the most important parameters for the infrared thermometer [7]. S is the distance from the MLX90614 to the bottle, and D is the diameter of the bottle. When use the instrument with the S/D equal 12/1, the distance coefficient requirement as shown in Table 4.

\subsection{Effects of Different Test Distances}

First, study the effects of different test distance on different materials. Plastic, ceramic, glass and paper are chosen as the test specimens, and the test distances $\mathrm{S}$ are at $5 \mathrm{~mm}$, $10 \mathrm{~mm}$ and $15 \mathrm{~mm}$ respectively.

The comparison of the measured temperature to the actual temperature of the commercially available plastic water bottle at different distance is shown in Fig. (12).

The comparison of the measured temperature to the actual temperature of the ceramic bottle at different distances is shown in Fig. (13).
The comparison of the measured temperature to the actual temperature of the glass bottle at different distances is shown in Fig. (14).

The comparison of the measured temperature to the actual temperature of the paper bottle at different distances is shown in Fig. (15).

The test result shows that the distance of the MLX90614 to the bottle will affect the measurement. The error is minimal while the distance $\mathrm{S}$ measured is at $5 \mathrm{~mm}$. With the distance $\mathrm{S}$ increase, the measurement error will also increase.

The maximal error are $2.6^{\circ} \mathrm{C}(\mathrm{S}=5 \mathrm{~mm}), 2.8^{\circ} \mathrm{C}(\mathrm{S}=10 \mathrm{~mm})$ and $3.1^{\circ} \mathrm{C}(\mathrm{S}=15 \mathrm{~mm})$ for glass bottle at $40^{\circ} \mathrm{C}$.

\subsection{Different Materials of the Bottle}

The material of bottles affects the measured results. We study the different results of bottles with different materials at a fixed distance.

The comparison of the measured temperature to the actual temperature of the bottles with different materials at a distance of $\mathrm{S}=5 \mathrm{~mm}$ is shown in Fig. (16).

The maximal error is $26^{\circ} \mathrm{C}$ at $40^{\circ} \mathrm{C}$ for glass and ceramic bottles at a distance of $\mathrm{S}=5 \mathrm{~mm}$.

The comparison of the measured temperature to the actual temperature of the bottles with different materials at a distance of $S=10 \mathrm{~mm}$ is shown in Fig. (17). 


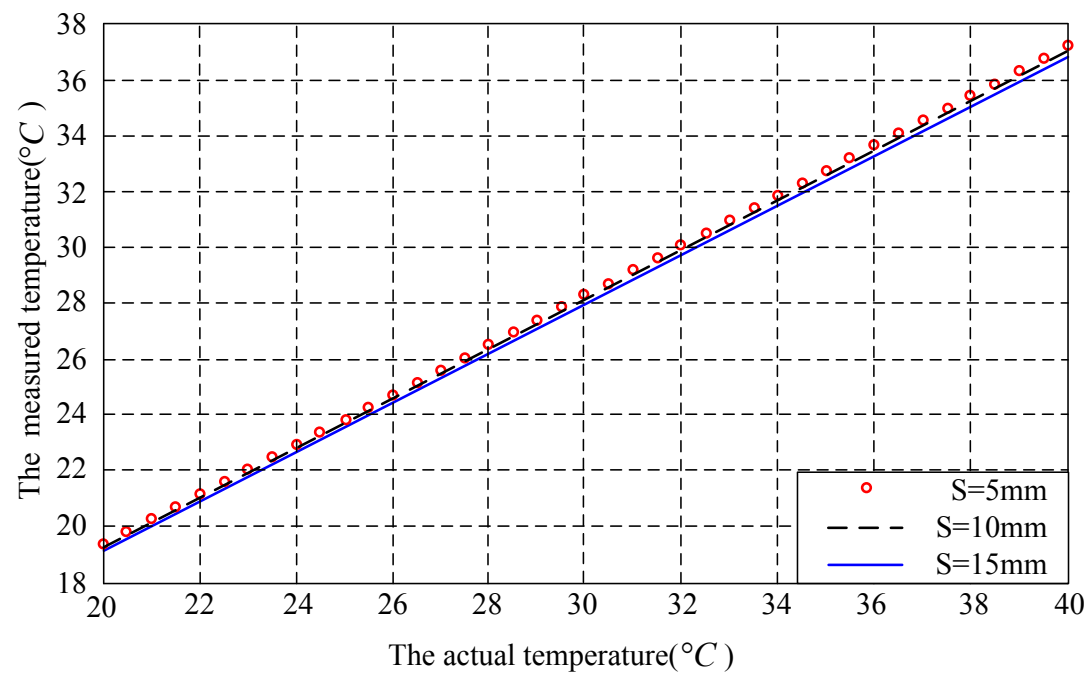

Fig. (13). The comparison of the measured temperature to the actual temperature of the ceramic bottle at different distances.

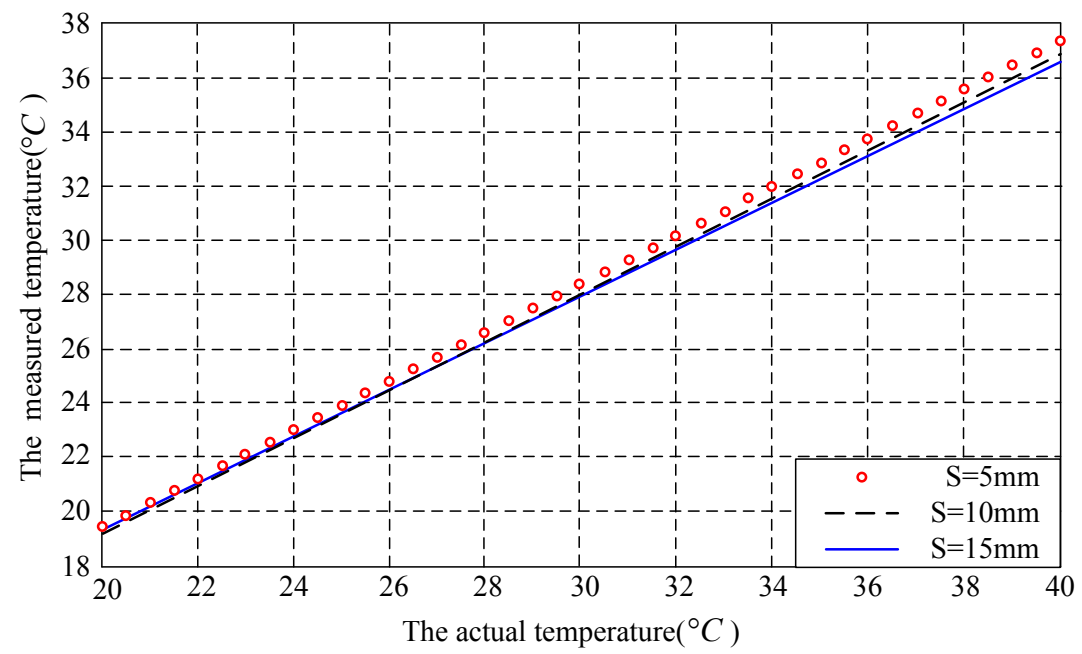

Fig. (14). The comparison of the measured temperature to the actual temperature of the glass bottle at different distances.

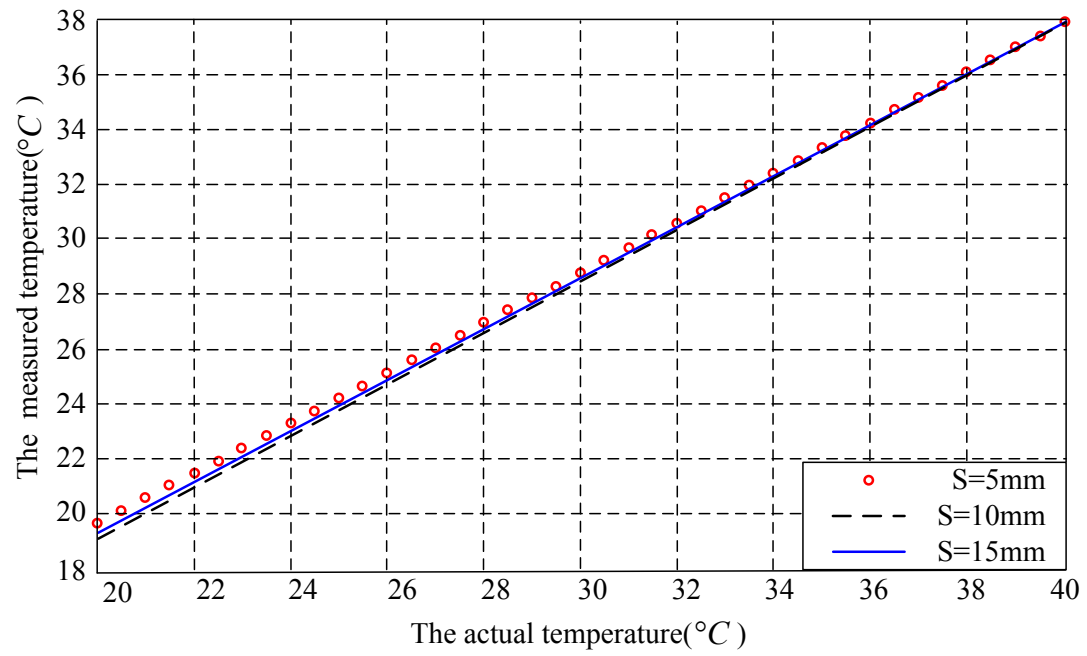

Fig. (15). The comparison of the measured temperature to the actual temperature of the paper bottle at different distances. 


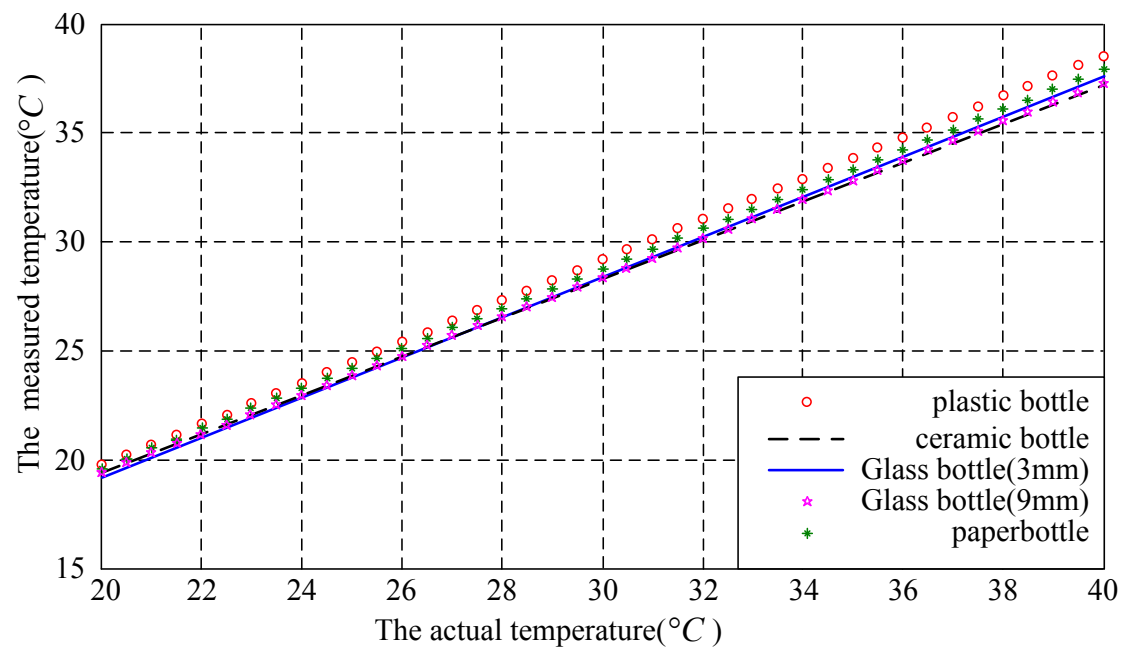

Fig. (16). The comparison of the measured temperature to the actual temperature of the bottles with different materials at a distance of $\mathrm{S}=5 \mathrm{~mm}$.

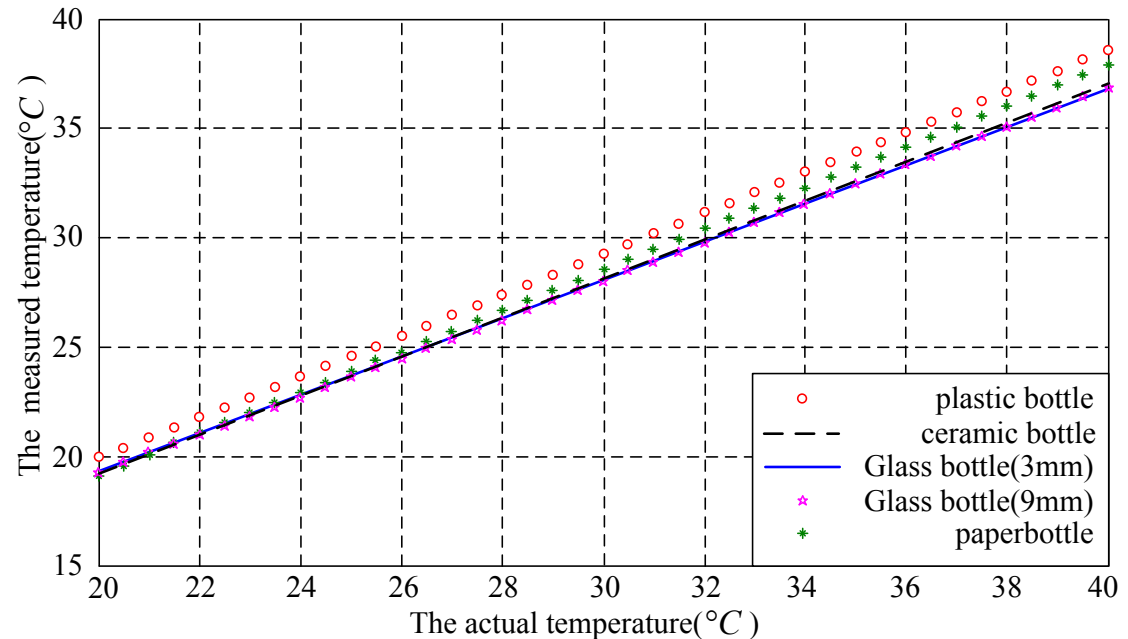

Fig. (17). The comparison of the measured temperature to the actual temperature of the bottles with different materials at a distance of $\mathrm{S}=10 \mathrm{~mm}$.

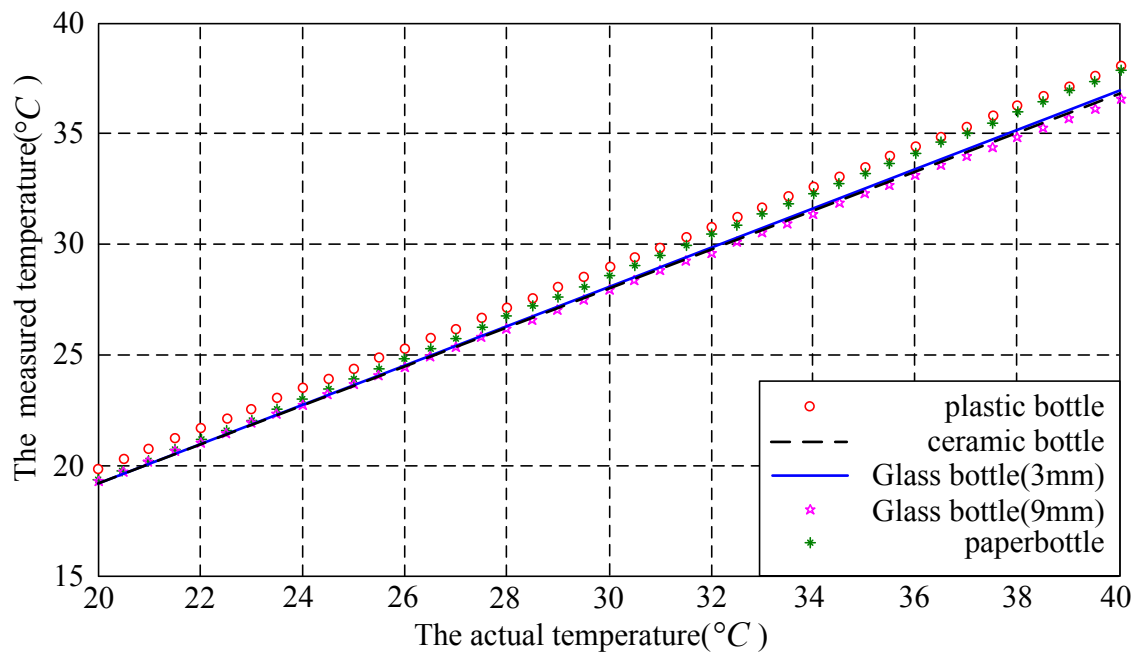

Fig. (18). The comparison of the measured temperature to the actual temperature of the bottles with different materials at a distance of $\mathrm{S}=15 \mathrm{~mm}$. 


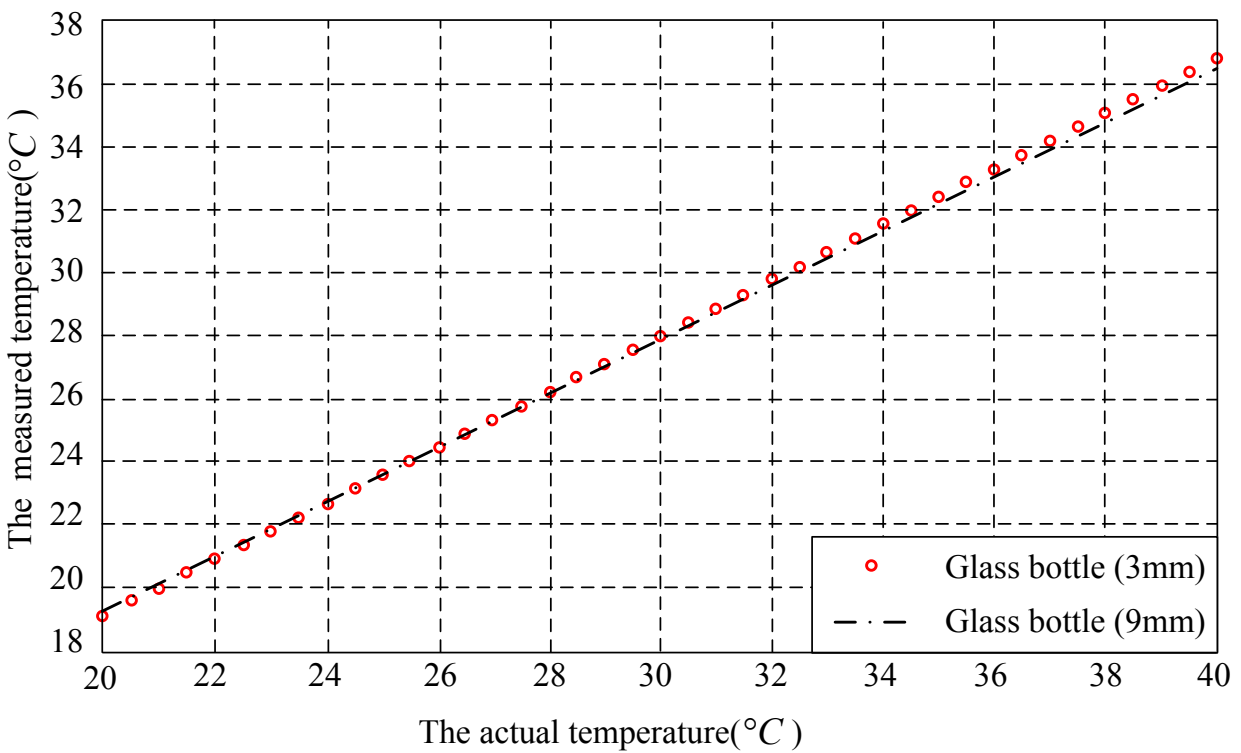

Fig. (19). The comparison of the measured temperature to the actual temperature of different wall thickness of the bottle at a distance of $\mathrm{S}=5 \mathrm{~mm}$.

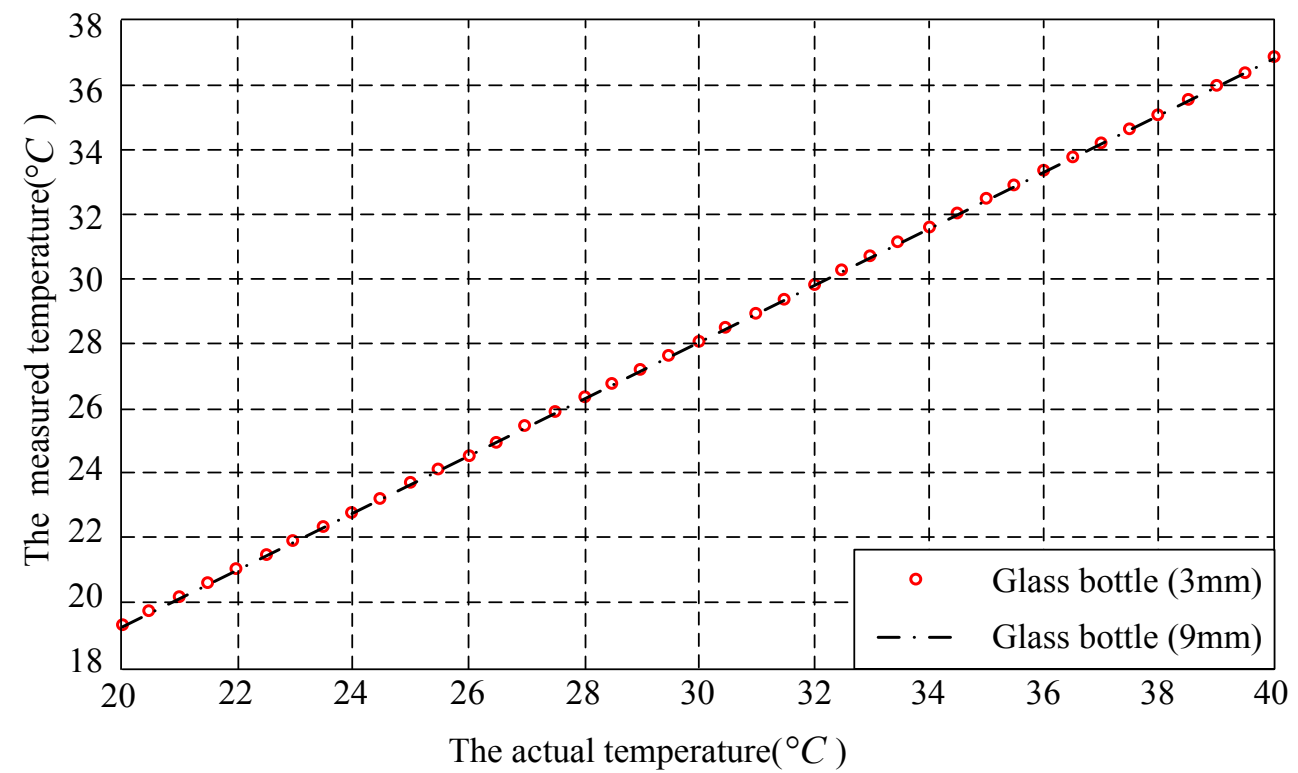

Fig. (20). The comparison of the measured temperature to the actual temperature of different wall thickness of the bottle at a distance of $\mathrm{S}=10 \mathrm{~mm}$.

The maximal error is $3.2^{\circ} \mathrm{C}$ at $40^{\circ} \mathrm{C}$ for the bottles with thin glass wall at a distance of $\mathrm{S}=10 \mathrm{~mm}$.

The comparison of the measured temperature to the actual temperature of the bottles with different materials at a distance of $\mathrm{S}=15 \mathrm{~mm}$ is shown in Fig. (18).

The maximal error is $3.2^{\circ} \mathrm{C}$ at $40^{\circ} \mathrm{C}$ for the bottles with thick glass walls at a distance of $\mathrm{S}=15 \mathrm{~mm}$.

The test results show that the bottles with different materials give different measurement errors. The commercially available plastic water bottle produces minimum error as compared to the other bottles, and the glass bottle produces the maximal error.

\subsection{Different Wall Thickness of the Bottle}

The wall thickness of bottles will affect the measured results too. We study the results of different wall thickness of glass bottles at a fixed distance.

The comparison of the measured temperature to the actual temperature of different wall thickness of the bottle at a distance of $S=5 \mathrm{~mm}$ is shown in Fig. (19).

The comparison of the measured temperature to the actual temperature of different wall thickness of the bottle at a distance of $\mathrm{S}=10 \mathrm{~mm}$ is shown in Fig. (20). 




Fig. (21). The comparison of the measured temperature to the actual temperature of different wall thickness of the bottle at a distance of $\mathrm{S}=15 \mathrm{~mm}$.

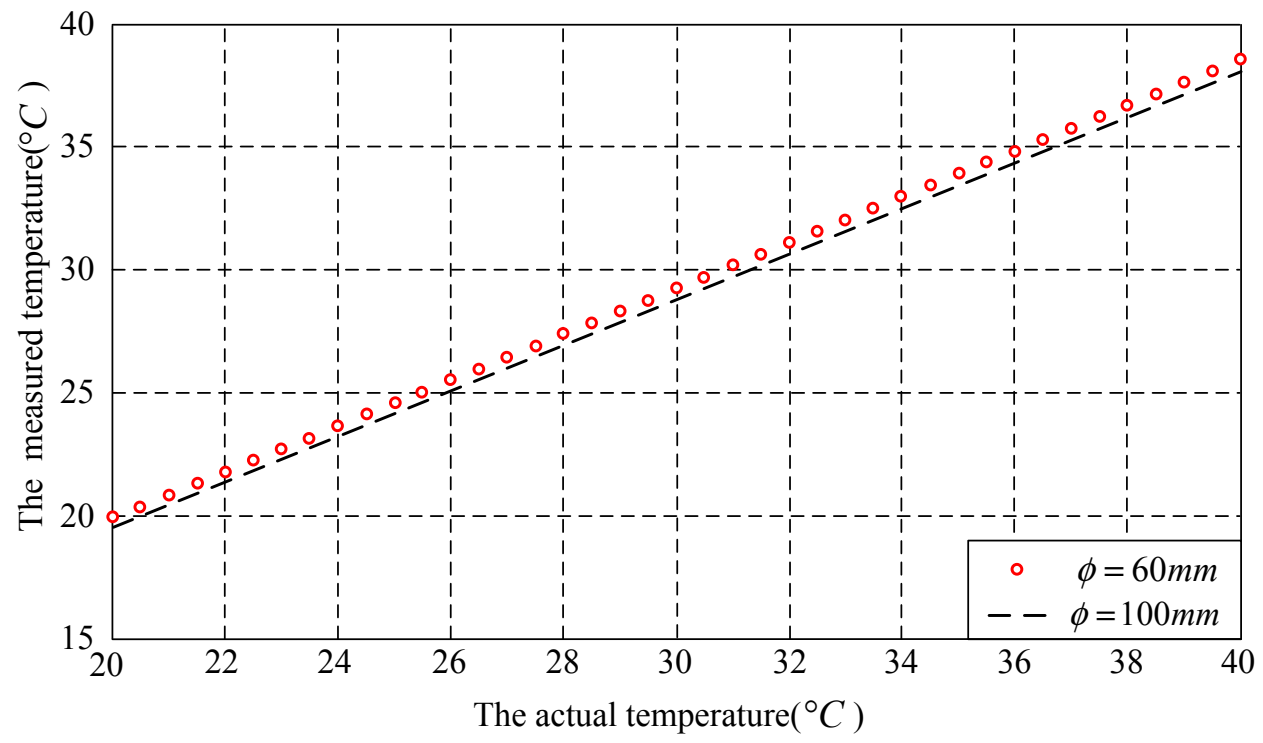

Fig. (22). The comparison of the measured temperature to the actual temperature of different diameters of the plastic bottle at a distance of $\mathrm{S}=10 \mathrm{~mm}$.

The comparison of the measured temperature to the actual temperature of different wall thickness of the bottle at a distance of $\mathrm{S}=15 \mathrm{~mm}$ is shown in Fig. (21).

The test results show that the different wall thickness of the bottles gives different measurement errors. Yet the maximal error of the thin-wall bottle and the thick-wall one is only $0.2^{\circ} \mathrm{C}$ between $20^{\circ} \mathrm{C}$ to $40^{\circ} \mathrm{C}$. The measurement errors are in the range of the design requirements, so the measurement errors can be ignored in different wall thickness of bottles.

\subsection{Different Sizes of the Bottle}

The diameter of the bottles may also affect the measured results. We study the different results of different diameters of commercially available plastic water bottle at a fixed distance.

The comparison of the measured temperature to the actual temperature of different diameters of the bottle at a distance of $\mathrm{S}=10 \mathrm{~mm}$ is shown in Fig. (22). 
The test results show that the different sizes of the bottles give different measurement errors. Yet the maximal error of the big plastic bottle and the small one is only $0.5^{\circ} \mathrm{C}$ at $40^{\circ} \mathrm{C}$ in the measured distance of $\mathrm{S}=10 \mathrm{~mm}$.

To reduce the measurement errors, we can choose the best test distance at $\mathrm{S}=5 \mathrm{~mm}$. The error can be controlled in the range of $0.5^{\circ} \mathrm{C}$ by adding temperature compensation algorithm according to the data of test.

\section{CONCLUSION}

The paper designed the non-contact Infra-Red thermometer based on the sensor of MLX90614 and the most favored microprocessor STM32F107 for the non-contact liquid security identification system. Completed the hardware circuit, made the PCB plate, programmed the software with Keil C and debugged by Keil uVision4 MDK V4.22. Then, we studied the effects of the results of the temperature measured by different bottles with different shapes, materials, sizes and wall thickness at various measuring distances. It meets the requirements of high reliability and low power consumption with real time response. The system works efficiently and meets the demands of the non-contact liquid security identification system.

\section{CONFLICT OF INTEREST}

The authors confirm that this article content has no conflict of interest.

\section{ACKNOWLEDGEMENTS}

This work was supported in part by the National "Twelve Five" Science and Technology Support Subproject (No. 2011BAK09B05) and National Natural Science Foundation of China (No. 61202399).

\section{REFERENCES}

[1] W.Y. Bei, and H. Jiang, "Study of Temperature Wireless Monitoring System Based on Infrared Technology", Comput. Measur. Control., no. 10, pp. 2397-2400, 2011.

[2] W.F. Liu, D.J. Song, J.C. Liu, and T.Han. "Design of an infrared thermometer based on STM32", Microcomputs Appl., vol. 31, no. 2, pp. 22-24, 2012.

[3] "STM32F107xx DataSheet", STMicroelectronics, 2009.

[4] "MLX90614 DataSheet", Melexis Corporation, 2007.

[5] T. $\mathrm{Li}$ and $\mathrm{Y}$. Hu, "Intelligent positioning portable thermometer", Transduc. Microsyst. Technol., vol. 33, no. 4, pp. 95-98, 2014.

[6] C.F. Sha. "MLX90614 IR Thermometer and Its Application", Modern Electron. Techniq., no. 22, pp. 36-40, 2007.

[7] S. M. Dai and G. R. Zhu, "The Analysis of the Error Caused From Infrared Temperature Surveying", J. SuZhou Instit. Silk Textile Technol., vol. 20, no. 1, pp. 25-29, 2000.

Received: September 16, 2014

(C) Jin et al.; Licensee Bentham Open.

This is an open access article licensed under the terms of the Creative Commons Attribution Non-Commercial License (http://creativecommons.org/licenses/ by-nc/4.0/) which permits unrestricted, non-commercial use, distribution and reproduction in any medium, provided the work is properly cited. 